\title{
DESIGN OF EXPERIMENTS PLATFORM FOR ONLINE SIMULATION MODEL VALIDATION AND PARAMETER UPDATING WITHIN DIGITAL TWINNING
}

\author{
MADHU SUDAN SAPKOTA ${ }^{1}$, EDWARD APEH ${ }^{1}$, MARK HADFIELD ${ }^{1}$, \\ ROBERT ADEY ${ }^{2}$ \& JOHN BAYNHAM ${ }^{2}$ \\ ${ }^{1}$ Faculty of Science and Technology, Bournemouth University Poole, UK \\ ${ }^{2} \mathrm{CM}$ BEASY Ltd., UK
}

\begin{abstract}
The process of developing a virtual replica of a physical asset usually involves using standardized parameter values to provide simulation of the physical asset. The parameters of the virtual replica are also continuously validated and updated over time in response to the physical asset's degradation and changing environmental conditions. The parametric calibration of the simulation models is usually made with trial-and-error using data obtained from manual survey readings of designated parts of the physical asset. Digital Twining (DT) has provided a means by which validating data from the physical asset can be obtained in near real time. However, the time-consuming process of calibrating the parameters so the simulation output of the virtual replica matches the data from physical asset persists. This is even more so when the calibration of the simulator is performed manually by analysing the data received from the physical system using expert knowledge. The manual process of applying domain knowledge to update the parameters is error prone due to incompleteness of the knowledge and inconsistency of the validation/calibration data. To address these shortcomings, an experimental platform implemented by integrating a simulator and a scientific software is proposed. The scientific software provides for the reading and visualisation of the simulation data, automation of the simulation running process and provide interface of the relevant validation and adaptive algorithmics. This comprehensive integrated platform provides an automated online model validation and adaptation environment. The proposed platform is demonstrated using BEASY - a simulator designed to predict protection provided by a cathodic protection (CP) system to an asset, with MATLAB as the scientific software. The developed setup facilitates the task of model validation and adaptation of the CP model by automating the process within a DT ecosystem.
\end{abstract}

Keywords: model adaptation, Digital Twin, cathodic protection, BEASY, software integration.

\section{INTRODUCTION}

In undertaking the structural health monitoring task, engineers use simulation tools to predict the risk to the structure from degradation mechanisms such as corrosion and cracking. More often, the parametric simulation models derived from the simulators on calibration are used to predict the location of the damage and its severity. Parameters as the model independent variables are model's input describing the properties of the materials and the environmental conditions the structure experiences. These model input variables cannot be directly measured due to structural complexity in most of the situations. Parameter setting for the model during realisation of a virtual replica of an existing physical structure thus relies upon the structural response-data obtained from sensors or survey. During such parametric calibration, the best set of parameters are found by correlating the model output to the available measurements from the physical system [1].

The traditional approach of calibration when performance validating data are available from the structure is based on a trial-and-error procedure involving manual iterative analyses and modifications. This task is normally performed by the engineers using their experience to choose the appropriate values until good correspondence is obtained (Fig. 1). As the parameters change with time, the process is repeated over the life of the structure based upon 
the requirement determined by expert and availability of the dataset. This results in significant computational efforts when performing model calibration/updating for complex models and in a repetitive manner.

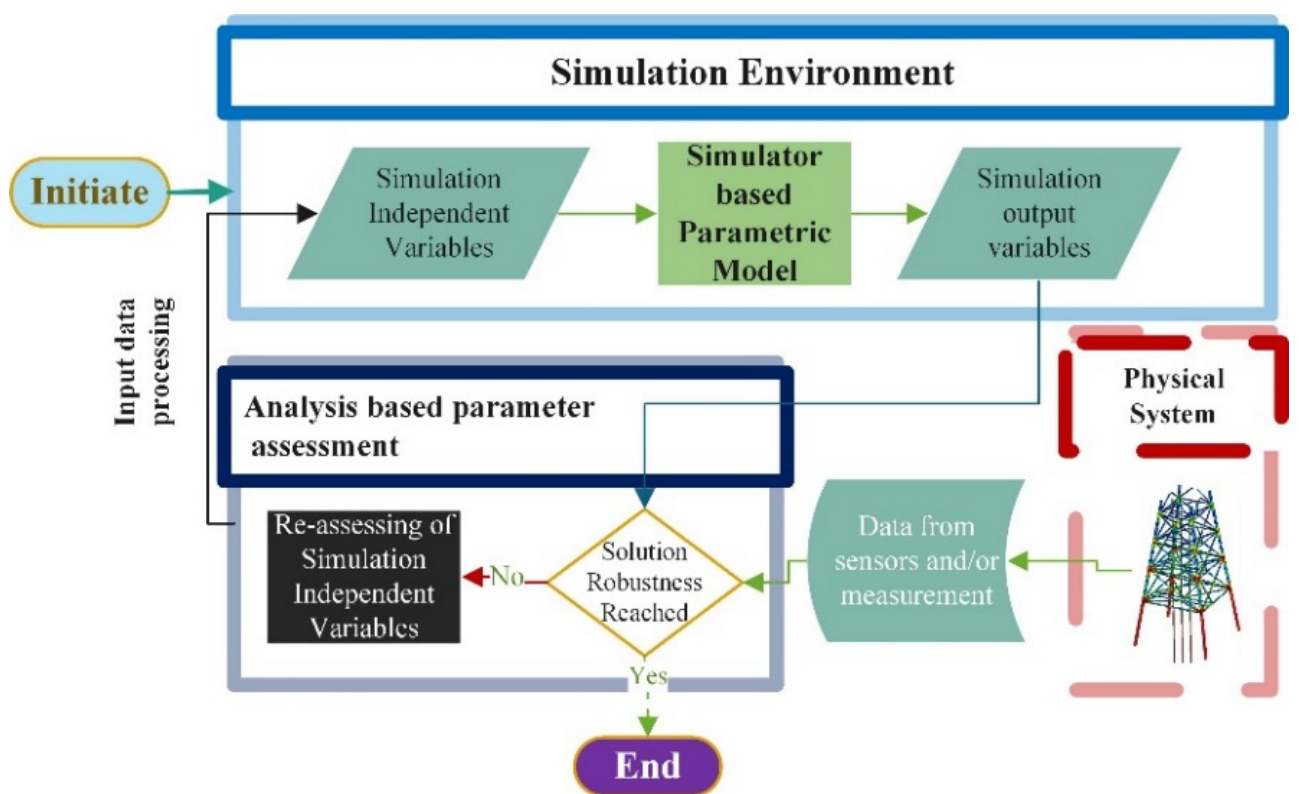

Figure 1: The parametric simulation model parameter updating for performance enhancement with output analysis (trial-and-error method).

The design optimisation [2] algorithms could be used to search the parameter space for the optimal set of parameters. However, the challenges like selecting the relevant optimisation algorithm, and implementing to the model still exist. These challenges are still relevant in the situations where commercial simulators are used for model building, but the platform for automated experimentation and analysis is absent. A platform that combines an optimisation software with a simulator can form a basis for decision-making for virtual replica [3]-[5]. Such an integration is more relevant and essential when two major tasks of modelling i.e. geometrical modelling (CAD design) and numerical simulation are provided by different software [6].

The virtual replica of an existing physical counterpart is recognised as Digital Twin (DT) in recent years. DT has been proposed with self-adaptive simulation characteristics and predictive capability [7]. Analytical supports to provide self-adaptation are anticipated incorporated within DT [8]. However, while enabling DT provided with the analytical features encapsulated within it, the features are not necessarily available under same software platform as for simulation. Therefore, the software integrated platform is still an important aspect for a DT model to enable a self-adaptive DT. The requisite of such experimental platform is to exploit the relevant features from available tools and/or software on enabling simulator-based Digital Twin.

This paper proposes software integration to have an experimental design platform required for continuous experimentation and analysis of the simulator-based prediction, 
while enabling and maintaining a DT. The supporting software representing the server of platform is either enriched with the analytical capability or analytical tool(s) interfacing capability, while simulating software is major tool for process simulation. This software integration platform provides a step towards establishing the core aspect of DT i.e. selfadaptation, leveraging the analytical aspects within DT.

\section{SIMULATOR BASED DIGITAL TWIN ENABLING}

\subsection{Simulator based modelling}

The use of previously validated model to the conceptual level assists in the design of not just one simulation model but many within the problem domain [9], [10]. The parametric simulation model building in multiple domains is already facilitated by the availability of commercial process simulators (simulating-software). Such simulators are generally based on current scientific understanding (physics of phenomena), often involving numerical approximation of differential equations, and they are implemented in complex computer programs [11]. The numerical approximation methods (e.g. Finite Element Method, Boundary Element Method) adopted for process simulation usually involves three major steps [12], [13]: (i) geometrical modelling of the structure(s), (ii) meshing of the geometry, and (iii) computational approximation of the Partial Differential Equation (PDE) solution. In tools supported modelling, steps (i) and (ii) are performed with CAD and meshing software tools, respectively, and then step (iii) requires a numerical solver. Commercially available simulators (e.g. AKSELOS, ANSYS, BEASY) provide the functionality of CAD modelling, meshing, as well as the behavioural process simulation i.e. numerical approximation of PDE.

When the commercially available simulator(s) provides the primary modelling requirements, the major efforts in realising virtual replica of physical asset are focused on determining model independent variables (parameters) such as material and environmental related parameters. The trial-and-error (Fig. 1) based traditional method during parametric calibration is inefficient and introduces time-delays due to manual performance, also has the drawbacks of not having the ability to calibrate multiple parameters simultaneously, and do not guarantee the best results. The simulation environment though enriched with core process simulation solver, thus requires analytical support that overcomes the drawbacks of traditional methods while tailoring the parametric model.

Experimental design, sensitivity analysis, and design (parameter) optimisation are widely adopted techniques within the systematic procedures of model performance enhancement [14]. Design optimisation is an approach that combines mathematical optimization algorithms with parametric simulation model to search the design (parameter) space for the optimal solution [2]. Gradient-based and non-gradient based algorithms are used during parameter optimisation [15], [16] for reaching the best fit. However, the challenges on selecting relevant techniques/algorithms still exists and remains time-consuming when selection and/with implementation to model is performed manually.

Automation of the analytical task by integrating online analytical environment to the simulation environment is the next step on addressing the pre-mentioned drawbacks. Correspondingly, the design optimisation functionality anticipated while determining best set of parameters can be facilitated using pre-available supporting tools (commercial-scientific software). 


\subsection{Digital Twin concept on modelling}

The DT concept is a new paradigm of interest in the field of modelling and simulation having online simulation as a core functionality of the system employing seamless assistance [17]. The definitions on DT are evolving rapidly in recent years as more insights are added to its features/potentials. Henceforward, DT is being preferred over the model in the context of adaptive simulation [18]. Moreover, the aspects of self-parameterisation and self-adaptation allowing DT to resemble its counterpart physical twin are of high importance to realize the potential of artificial intelligence (AI) within DT [8], [19]. Also, a digital twin concept is not only about a single behavioural simulation, mostly involved multiple process co-simulations representing different behavioural phenomena. This highlights the DT as collaborative models to provide a comprehensive representation of the system's simultaneously occurring multiple dynamic phenomena. Going along with the concepts on DT, while enabling DT as high-fidelity simulation model provided with self-analysis features or DT with cosimulations, software integration [7] is anticipated.

Despite the limitations of not having standardized concept on DT, one can reap the benefits that the DT could provide, like Digital Twinning on having validating data from the physical asset in near real time. Thus, software integration requisite for model performance enhancement is preferably undertaken within Digital Twin concept, not only to utilise the benefits DT provides, but also to standardise the DT's potential(s) and requirements.

\section{DESIGN OF EXPERIMENTS PLATFORM WITH SOFTWARE INTEGRATION}

The implementation of open-source or commercial software (tools) provides the analytical assistance during model calibration/adaptation i.e. implementing adaptive algorithms to the parametric models [3]-[5]. Such supporting tools when used in collaborative workflows with the model makes the simulation easier to compile, test, analyse, and suggest changes to model's input. On the other hand, adopting DT concept means incorporating features like potential to handle data, perform experimentation, and implement adaptive algorithm together with simulator to have a robust predictive tool. For this, experimental platform providing the integration between the major tool (simulator(s)) and the supporting tool(s), with automated data and control signal flow between them is proposed within Digital Twinning concept.

The scientific software should either include tool(s) (Fig. 2) or at least provide the interface to the external tool(s) for analytical supports including design optimisation. This support then would be utilised within the platform where scientific software (server) serves the simulator as its client [20]. Before this, the server has the role of input-output data management like filtering and data mapping, preparing dataset for the simulator and the analytical tool(s). Towards, analytical aid on model performance enhancement, it provides a design of experiments platform for experimentation and analysis using designated internal/externally interfaced algorithms. The server software similarly provides the opportunity for the visualisation of the data to denote the state and discrepancies in the performance of the model from the actual system. Likewise, automated data flow between the platform and the data-server allows retro-perspective analysis when corresponding timeseries data are available into the data server. In addition, the platform should provide model performance validating criterion/algorithm separately or integrated to the platform, as adaptation task cannot be carried out without validation task synchronised [21].

While enabling DT and/or maintaining it over the lifetime, the scientific software-based integrated platform forms the basis of automation of the parameter updating required for model performance validation and enhancement. 


\section{CASE STUDY: BEASY BASED CATHODIC PROTECTION MODELLING AND ITS ENHANCEMENT}

\subsection{Background}

Cathodic protection (CP) is most frequently used for the protection of underground or underwater (sea water) metallic infrastructures from corrosion. The design of the $\mathrm{CP}$ system depends upon assumptions (often referred to as design rules) about the performance of other protection measures such as coatings, in particular the rate at which coatings are to be assumed to degrade over the life of the structure. The performance of the CP system can be evaluated and optimised based on the design rules using a CP simulation model which predicts year by year the protection potentials, the depletion of the anodes, and in the case of Impressed-Current-Cathodic-Protection (ICCP), the current to be required by the system over its life [22].

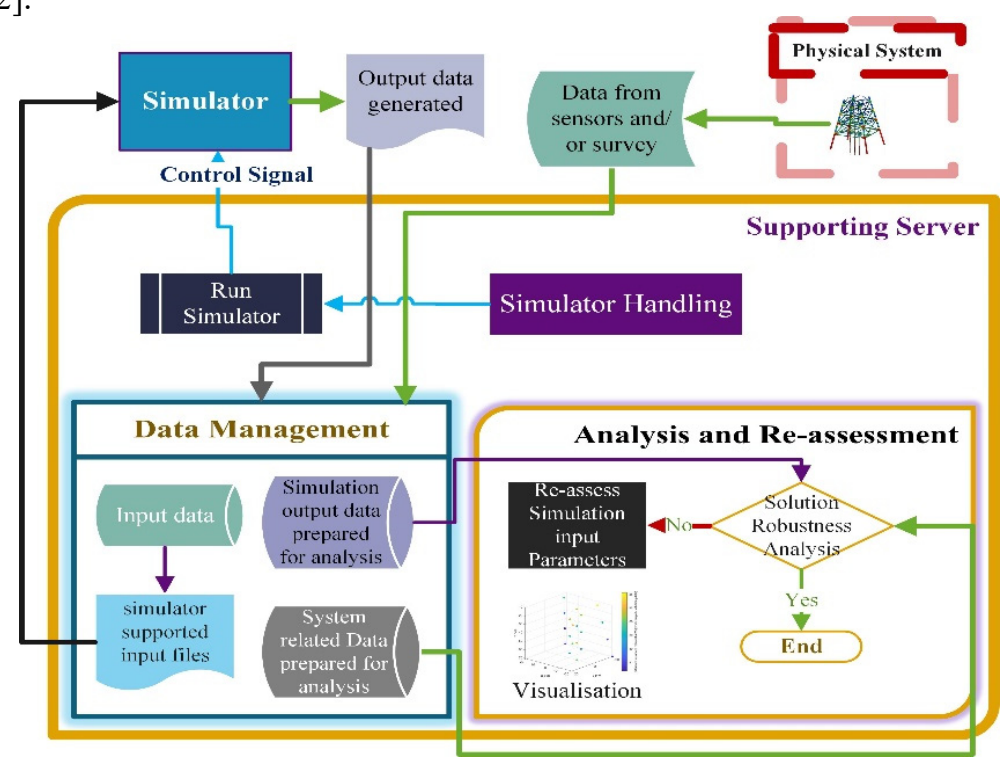

Figure 2: Integration of server and simulator for automation of the process of data management and analytical support required on simulator-based DT enabling.

While this type of simulation provides valuable information to the design engineer by confirming that the required protection will be achieved. In reality, the actual performance of the CP system will be often different as coatings for example often degrade at different rates to that described in the design rules, environmental conditions may vary, the "as-built" structure may be different and changes and retrofits are made over time. Integrating the CP data collected during the routine inspection surveys with a CP simulation model on calibrating/adapting the model to match the inspection data enables a "digital twin" of the structure [23]. In this way, the digital twin represents the behaviour of the structure and the $\mathrm{CP}$ system at the time the inspection survey was performed. This then provides the ability to predict the present and future protection for all parts of the structure.

By repeating the process with each new inspection report the engineer can monitor the differences between the model predictions and survey data systematically to assess current 
"health" of the structure, identify anomalies, predict and plan for future risks, optimise the inspection strategy and provide early identification of problems which will require actions.

\subsection{BEASY as a simulator for cathodic protection model}

BEASY tool - a commercial parametric simulator designed to simulate the behaviour of galvanic corrosion problems and cathodic protection designs is adopted to represent a virtual replica of a CP system.

The CP simulation model is based on the prediction of the distribution of electrical potential and protection current density on the electrode surfaces as well as at the corresponding points. The calculation of electric potential and current density distribution is based on the solution of the well-known Laplace partial differential eqn (1).

With assumption that electrolyte is homogeneous

$$
-\nabla(k \nabla \varphi)=0
$$

where $k=$ electric conductivity, $\varphi=$ electric potential and $\nabla$ is Nabla operator.

BEASY-CP provides numerical approximation of Laplace's equation obtained with Boundary Element Method (BEM) [11], [22] for steady state corrosion. Also, commercial BEASY tool facilitates on geometrical modelling and meshing. The data about geometry, meshing, and materials and surrounding related parameters data can be exported to text files and feed to the solver for numerical approximation (Fig. 3).

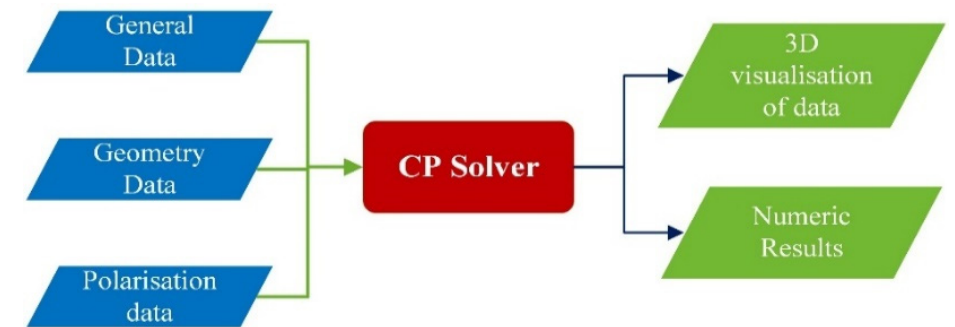

Figure 3: Inputs-outputs for BEASY tool based cathodic protection simulation.

The numeric results provided by the simulation are analysed with data collected during the routine inspection surveys of physical CP system. Calibration is made with repetitive analysis and updating of the input parameter related value into the input supported files before to run next simulation and get new data for analysis.

A CP simulation model of a marine structure (Fig. 4) protected by sacrificial anodes was built using the BEASY tool. The model parameters required by the cathodic protection model for the CP system of structure (Fig. 4) are:

- Polarisation behaviour: The relationship between potential and current density represents the electrode kinetics of the metal in the seawater. It provides the boundary condition while solving the numerical problem.

- Polarisation curve for Material 1 of the structure (Fig. 4).

○ Polarisation curve for Material 2 of the structure (Fig. 4).

- Conductivity/resistivity: Surrounding medium/material related.

- Sea water related conductivity $($ Siemen $/ \mathrm{m})$.

- Sea-bed related conductivity $($ Siemen $/ \mathrm{m})$. 


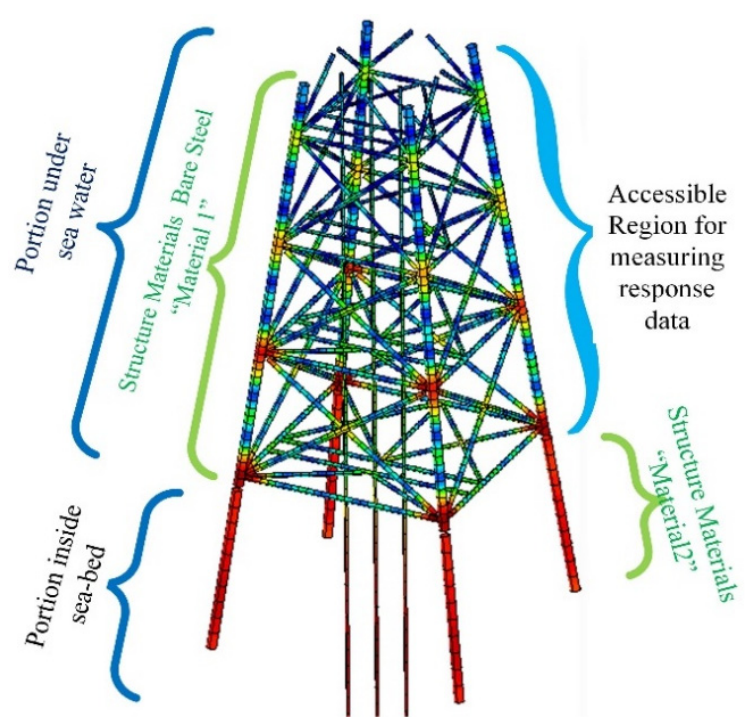

Figure 4: The project adopted geometrical model of marine structure protected with sacrificial anodes [21].

As the polarisation curves for representing the polarisation behaviour are graphical representation (Fig. 5) and dynamics with time, a quantitative representation should be established for readjustment of the polarisation data. To address the possible change in polarisation behaviour, the curve transformation value (expanding or squeezing factor) is taken as a variable (parameter) keeping the curve constant obtained from design rule. This parameterisation concept can be understood as a modification of the diffusion limiting current in the polarisation behaviour of the materials involved. The transformation vector or parameter is termed as "p-value" in this case study.

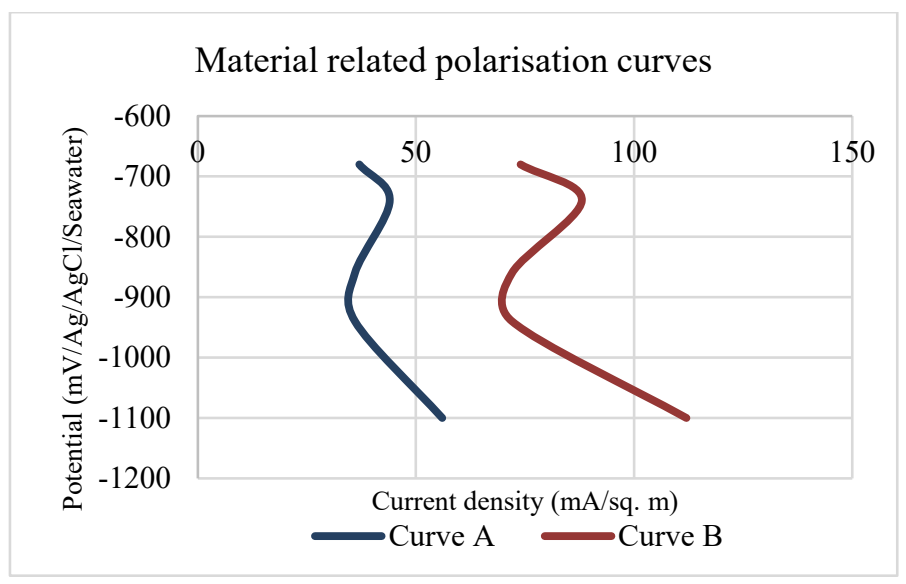

Figure 5: Two different polarisation curves that can be transformed from one to other with some transformation value [21]. 


\subsection{Software integration with MATLAB [24] as a server}

Even though the simulation task is mostly dependent upon the simulator, the scientific software in the design of experiments platform remains as the server. The extensive data analysis, plotting capability and the availability of different optimisation algorithm, with the MATLAB enables completion of assess-modify-check loops in reduced computational time. Optimisation tool within MATLAB such as "fmincon" and "fminunc" [24] likewise can be used for constrained and unconstrained optimisation problem respectively. MATLAB - a scientific software granted with these qualities is selected as the Server for the integration platform to enable DT for a CP system.

Furthermore, data reading and modifying from/to the input-output files to the simulation solver can be performed with the server software or using other open-source tools (e.g. python based). MATLAB as server also provides the integrating ability to such tools. In this case study, python-based codes were incorporated to facilitate the data transfer and update in files shared between server and the simulator.

\subsection{Tools integration for optimisation based parameter updating and analysis}

\subsubsection{Tool for optimisation-based parameter calibration/adaptation}

For the task of adaptation of the CP model with parameter fitting, a gradient based algorithm "quasi-newton" is chosen. The reason for choosing gradient based approach as opposed to other optimisation techniques, such as genetic algorithms or neural networks, is that the problem space of CP model is mostly monotonic. Optimised values of parameters can, therefore, be found quickly with gradient-based algorithms, i.e. in a lower iteration(laps) count and consequently in a lower amount of time. On this basis, unconstrained optimisation tool "fminunc" within MATLAB is utilised and supported with "quasi-newton" [20] algorithm.

\subsubsection{CP model's performance validating data}

The data types that can be practically obtained from the structure and from the $\mathrm{CP}$ model simulation run as well are: (a) surface potential $(\mathrm{mV})$, (b) normal current density $\left(\mathrm{mA} / \mathrm{m}^{2}\right)$ and (c) electric field $(\mathrm{mV} / \mathrm{m})$ [22]. However, the data dependency for validation depends upon the complexity of the model.

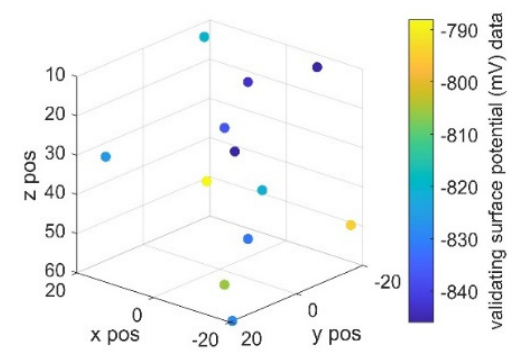

(a)

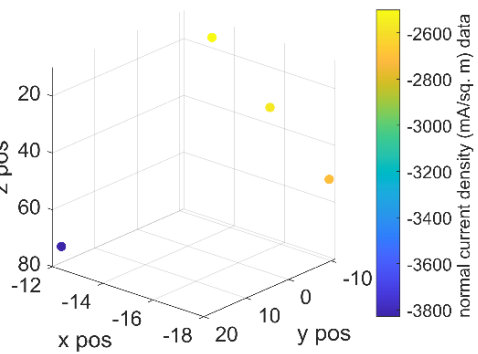

(b)

Figure 6: The validation data obtained after simulation run from the reference model. (a) Surface potential; and (b) Normal current density, from selected validating position of structure's surface (Fig. 4). 
At this stage, validating data are generated from a virtual reference model provided with different parameter values considering the possible polarisation behaviour in future than to the parameter values set for the initial primary model whose value(s) mostly depends upon the design rules. Two types of validating data are considered surface potential $(\mathrm{mV})$ and normal current density $\left(\mathrm{mA} / \mathrm{m}^{2}\right)$ and the validating data positions count are 12 and 4 respectively from the structure's surface (Fig. 6).

\subsubsection{Objective function for optimisation problem}

Reducing the discrepancies between the validating and response data from simulation is the goal of optimisation. Assuming two different validating datatypes (Section 4.4.2), Normalised Mean Square difference between validating and model output data with weightage constant $(2: 1)$ provided for both data types, is used as an Objective function.

\subsubsection{Initial stage primary model and parameters value}

The highly sensitive parameters are the focus of in this case study, which is why the other parameters' values are kept constant. It is easily obtained from sensitivity analysis that the parameters more sensitive to response data are the "p-value of Material 1 related polarisation curve" and "Sea-water conductivity".

Table 1: Parameter's value provided to the initial primary model presumably from design data rules.

\begin{tabular}{|c|c|}
\hline "Material 1" polarisation curve p-value & Sea-water conductivity \\
\hline 1.7500 & 3.0000 \\
\hline
\end{tabular}

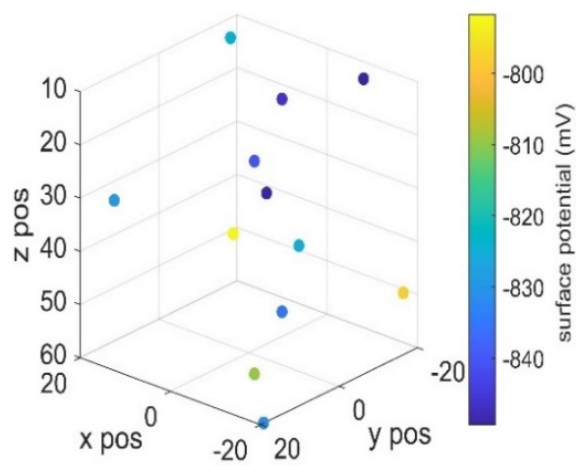

(a)

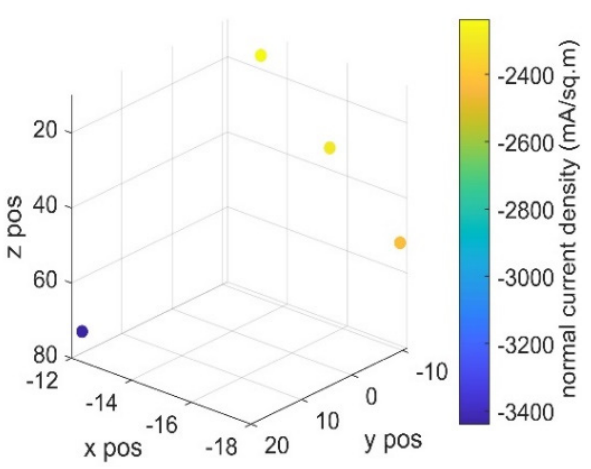

(b)

Figure 7: The response surface data. (a) Surface potential; and (b) Current density for the corresponding positions of the validating data in Fig. 6. Obtained after simulation run of initial primary model with parameters value given in Table 1.

\subsubsection{Optimisation performance analysis}

An initial parametric CP model with provided parameter values as in Table 1 which results in response data as in Fig. 7 on simulation run is considered for optimisation-based parameter updating. Validation data (Section 4.4.2), metric (Section 4.4.3) and optimisation algorithm 
(Section 4.4.1) are considered for the optimisation requirement and is performed within the MATLAB based platform.

The discrepancies between the validating data and the model output (initial model and solution model) can be visualised from the Fig. 8, while the optimisation step related insight can be obtained from Table 2, both are generated within the supporting software (MATLAB). The optimisation based parametric search ends when the optimisation-algorithm cannot decrease the objective function further, in the search direction.
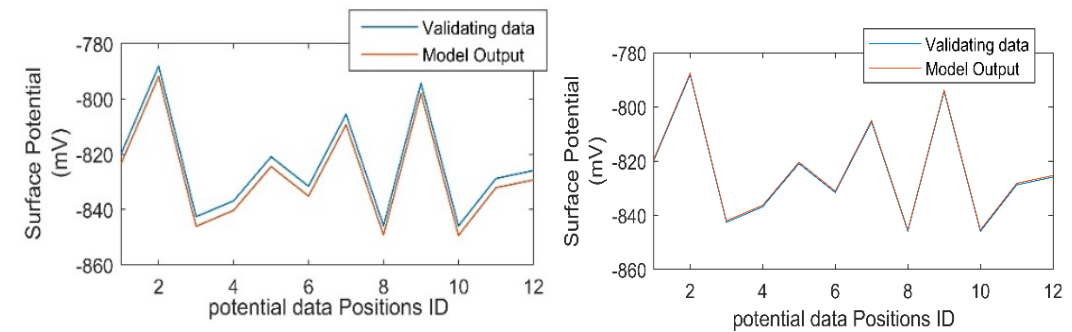

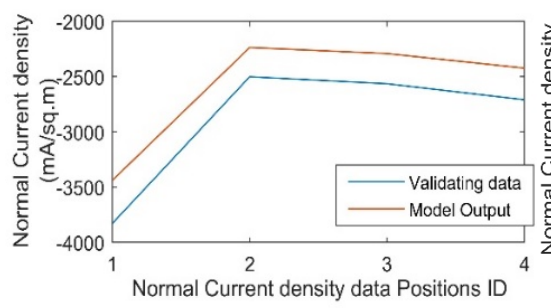

(a)

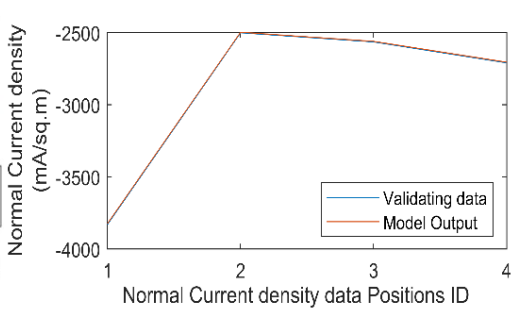

(b)

Figure 8: Discrepancies plot between model output and the validating data. (a) Initial primary model; and (b) Solution model after optimisation-based parameter updating.

Table 2: Model parameter stages during the optimisation problem before reaching to a solution.

\begin{tabular}{|c|c|c|c|c|}
\hline Iteration & F-count & Material 1 p_value & $\begin{array}{c}\text { Sea-water } \\
\text { conductivity }\end{array}$ & $\begin{array}{c}\text { Objective function } \\
(\mathrm{F}(\mathrm{x}))\end{array}$ \\
\hline 0 & 3 & 1.7500 & 3.0000 & $5.5168 \mathrm{e}-03$ \\
\hline 1 & 9 & 1.8145 & 3.0443 & $3.5445 \mathrm{e}-03$ \\
\hline 2 & 12 & 2.0516 & 3.2340 & $3.5730 \mathrm{e}-05$ \\
\hline 3 & 15 & 2.0515 & 3.2422 & $2.8556 \mathrm{e}-05$ \\
\hline 4 & 18 & 2.0425 & 3.2655 & $1.5382 \mathrm{e}-05$ \\
\hline 5 & 21 & 2.0229 & 3.2959 & $4.5574 \mathrm{e}-06$ \\
\hline 6 & 24 & 2.0027 & 3.3186 & $1.5651 \mathrm{e}-06$ \\
\hline
\end{tabular}

In the given case, it takes a total of six iterations excluding the initial one to converge to the best set of parameter values. Similarly, counts for repetitive objective function calculation are represented as F-count (Table 2) which is also equivalent to the total data exchange count between server and the simulation, and the total count of simulation runs. 
This parameter updating which could take many hours or days when performed manually, is reduced to less than a few hours including all the simulation running time. The importance of the platform is highlighted by the significant reduction of model calibrating time using the automated experimental platform than compared to the manual approach.

\section{CONCLUSION}

This paper discussed the design of experiments platform for simulation performance enhancement while realising a Digital Twin of a physical asset. The scientific software-based integrated platform forms the basis of automation on enabling virtual replica (Digital Twin) from the pre-available simulator(s), utilising supports from the analytical tool(s).

The case study shows the application of the integration platform to achieve a cathodic protection Digital Twin. Optimisation procedures were undertaken using the approach, and automatic parameter updating of a $\mathrm{CP}$ model to calibrate the model with the measured data has been demonstrated.

Overall, the proposed approach combines advantages offered by scientific and commercial software(s) to have a comprehensive tool in reduced time capable to predict the present and future health of a structure. This approach however is generic and can be implemented beyond the corrosion and cathodic protection domain.

Further work will be focused on the automation of the overall customised systematic procedures on enabling DT, besides the optimisation task.

\section{ACKNOWLEDGEMENT}

This work has been undertaken as part of a match-funded $\mathrm{PhD}$ research between Computational Mechanics International Limited and Bournemouth University, UK.

\section{REFERENCES}

[1] Oliveira, H.L. \& Leonel, E.D., Constitutive relation error formalism applied to the solution of inverse problems using the BEM. Engineering Analysis with Boundary Elements, 108, pp. 30-40, 2019.

[2] Papalambros, P.Y. \& Wilde, D.J., Principles of Optimal Design: Modeling and Computation, Cambridge University Press, 2000.

[3] Mitchell, M., Udugama, I., Currie, J. \& Yu, W., Software integration for online dynamic simulation applications. 2017 6th International Symposium on Advanced Control of Industrial Processes (AdCONIP), IEEE, pp. 360-364, 2017.

DOI: 10.1109/ADCONIP.2017.7983807.

[4] Borodin, V., Bourtembourg, J., Hnaien, F. \& Labadie, N., COTS software integration for simulation optimization coupling: Case of ARENA and CPLEX products. International Journal of Modelling and Simulation, 39(3), pp. 178-189, 2019.

DOI: 10.1080/02286203.2018.1547814.

[5] Benaouali, A. \& Kachel, S., Multidisciplinary design optimization of aircraft wing using commercial software integration. Aerospace Science and Technology, 92, pp. 766-776, 1 Sep. 2019. DOI: 10.1016/j.ast.2019.06.040.

[6] Whitfield, R.I., Duffy, A.H., Gatchell, S., Marzi, J. \& Wang, W., A collaborative platform for integrating and optimising computational fluid dynamics analysis requests. Computer-Aided Design, 44(3), pp. 224-240, 2012.

[7] Rasheed, A., San, O. \& Kvamsdal, T., Digital twin: Values, challenges and enablers. arXiv preprint arXiv: 1910.01719, 2019. 
[8] Barricelli, B.R., Casiraghi, E. \& Fogli, D., A survey on digital twin: Definitions, characteristics, applications, and design implications. IEEE Access, 7(Ml), pp. 167653-167671, 2019. DOI: 10.1109/ACCESS.2019.2953499.

[9] Abdelmegid, M.A., González, V.A., O’Sullivan, M., Walker, C.G., Poshdar, M. \& Ying, F., The roles of conceptual modelling in improving construction simulation studies: A comprehensive review. Advanced Engineering Informatics, 46(Sep.). DOI: 10.1016/j.aei.2020.101175.

[10] Robinson, S., Arbez, G., Birta, L.G., Tolk, A. \& Wagner, G., Conceptual modeling: Definition, purpose and benefits. 2015 Winter Simulation Conference (WSC), IEEE, pp. 2812-2826, 2015.

[11] Brynjarsdóttir, J. \& O’Hagan, A., Learning about physical parameters: The importance of model discrepancy. Inverse Problems, 30(11), p. 114007, 2014. DOI: $10.1088 / 0266-5611 / 30 / 11 / 114007$.

[12] Zienkiewicz, O.C., Taylor, R.L. \& Zhu, J.Z., The Finite Element Method: Its Basis and Fundamentals, Elsevier, 2005.

[13] Aliabadi, M.H., The Boundary Element Method: Applications in Solids and Structures, vol. 2, John Wiley and Sons, 2002.

[14] Law, A.M., Kelton, W.D. \& Kelton, W.D., Simulation Modeling and Analysis, McGraw-Hill: New York, 2000.

[15] Venter G., Review of optimization techniques. Encyclopedia of Aerospace Engineering, 2010.

[16] Whitley, D., Rana, S., Dzubera, J. \& Mathias, K.E., Evaluating evolutionary algorithms. Artificial Intelligence, 85(1-2), pp. 245-276, 1996.

[17] Boschert, S. \& Rosen, R., Digital twin: The simulation aspect. Mechatronic Futures, Springer: Cham, pp. 59-74, 2016.

[18] Wright, L. \& Davidson, S., How to tell the difference between a model and a digital twin. Advanced Modeling and Simulation in Engineering Sciences, 7(1), pp. 1-3, 2020. DOI: 10.1186/s40323-020-00147-4.

[19] Schleich, B., Answer, N., Mathieu, L. \& Wartzack, S., Shaping the digital twin for design and production engineering. CIRP Annals, 66(1), pp. 141-144, 2017.

[20] Inzillo, V., Santamaria, A.F. \& Quintana, A.A., Integration of Omnet++ simulator with Matlab for realizing an adaptive beamforming system. 2017 IEEE/ACM 21st International Symposium on Distributed Simulation and Real Time Applications (DSRT), IEEE, pp. 1-2, 2017.

[21] Sapkota, M.S., Apeh, E., Hadfield, M., Haratian, R., Aey, R. \& Baynham, J., An approach for adaptive model performance validation within digital twinning. CMEM21, 2021.

[22] Adey, R.A., Modelling of Cathodic Protection Systems, WIT Press: Southampton and Boston, 2006.

[23] Adey, R., Peratta, C. \& Baynham, J., Corrosion data management using 3D visualisation and a digital twin. NACE International Corrosion Conference Proceedings, NACE International, pp. 1-13, 2020.

[24] MATLAB and Statistics Toolbox Release 2012b, The MathWorks, Inc.: Natick, MA. 